\title{
QSAR Study for Carcinogenic Potency of Aromatic Amines Based on GEP and MLPs
}

\author{
Fucheng Song ${ }^{1}$, Anling Zhang ${ }^{2}$, Hui Liang ${ }^{1}$, Lianhua Cui ${ }^{1}$, Wenlian $\mathrm{Li}^{1}$, Hongzong $\mathrm{Si}^{3}{ }^{3} *$, \\ Yunbo Duan ${ }^{3}$ and Honglin Zhai ${ }^{4}$ \\ 1 Department of Public Health, Qingdao University Medical College, Qingdao 266071, China; \\ qdsongfucheng@126.com (F.S.); qdlianghui@126.com (H.L.); qdlhcui@163.com (L.C.); \\ lwenl27@163.com (W.L.) \\ 2 Modern Educational Technology Center, Qingdao University, Qingdao 266071, China; \\ anling_zhang@126.com \\ 3 Institute for Computational Science and Engineering, Laboratory of New Fibrous Materials and \\ Modern Textile, The Growing Base for State Key Laboratory, Qingdao University, Ningxia Road 308, \\ Qingdao 266071, China; bobduan@hotmail.com \\ 4 Department of Chemistry, Lanzhou University, Lanzhou 730000, China; zhaihl@163.com \\ * Correspondence: sihz03@126.com; Tel./Fax: +86-532-8595-0768
}

Academic Editor: William Chi-shing Cho

Received: 7 September 2016; Accepted: 24 October 2016; Published: 15 November 2016

\begin{abstract}
A new analysis strategy was used to classify the carcinogenicity of aromatic amines. The physical-chemical parameters are closely related to the carcinogenicity of compounds. Quantitative structure activity relationship (QSAR) is a method of predicting the carcinogenicity of aromatic amine, which can reveal the relationship between carcinogenicity and physical-chemical parameters. This study accessed gene expression programming by APS software, the multilayer perceptrons by Weka software to predict the carcinogenicity of aromatic amines, respectively. All these methods relied on molecular descriptors calculated by CODESSA software and eight molecular descriptors were selected to build function equations. As a remarkable result, the accuracy of gene expression programming in training and test sets are 0.92 and 0.82 , the accuracy of multilayer perceptrons in training and test sets are 0.84 and 0.74 respectively. The precision of the gene expression programming is obviously superior to multilayer perceptrons both in training set and test set. The QSAR application in the identification of carcinogenic compounds is a high efficiency method.
\end{abstract}

Keywords: QSAR; aromatic amines; gene expression programming; multilayer perceptrons

\section{Introduction}

Aromatic amines (AAs) are indispensable material in the process of synthesis azo colorants, which have strong tinting strength, bright color, and durability. So, the azo colorants have been widely applied to textile industry, food additives, cosmetics, and plastics [1,2]. In our life environment, we can come into contact with AAs at any time, such as gorgeous clothes, colorful food, and polluted air and water. The main ways of AAs to enter the body are the skins contact and digestive tract [3]. It is recognized that some AAs be verified or be suspected as human carcinogens. The enzyme P450 can help AAs convert into arylnitreniumlons in the body, which combine with C8 position of guanine in DAN.

Through extended exposure to the compounds, the structure of the DNA will be changed and a malignant tumor will appear. As a result, it leads to bladder, ureteral, renal, and pelvic carcinoma and other malignant diseases [4-6]. The European Commission Regulation 552/2009/EC has banned carcinogenic AAs to be used in textile and leather articles [7]. With the rapid development of chemical 
industry, a large number of compounds are produced and used. Compounds eventually are distributed in the environment by various uses, which strongly influence environmental and human health $[8,9]$.

Due to the high carcinogenicity of AAs, recognition of the toxicity and carcinogenicity of the new AAs has special significance in toxicology. Thus, it is very important to assess the security risk of compounds. However, it is a huge project to assay the large number of compounds by experimental means. Toxicity identification of new compounds is very harmful to experimental animals. Even some experiments violate ethics requirements [10]. So, it is necessary to develop a simple, fast, and available approach to measure the property of security risk of compounds. The quantitative structure activity relationship (QSAR) method not only can quickly establish a reliable predicting model, but also can reveal the damaging effect mechanism of the poison interacting with the body and provide the reference information of designing and synthesizing safer and eco-friendly real green compounds [11]. Study [12] has been carried out with the aid of a combined quantum mechanics/molecular mechanics (QM/MM) computations to explore the detoxifying mechanism of agGSTe2 toward DDT. In this thesis, all AAs were randomly divided into a training set and test set, and then we set up prediction models based on molecular descriptors of AAs.

In the last two decades, many scholars had solved prediction problems by the establishment of bionic mathematics calculation model and the achievements were surprising [13]. Establishing a stability and rapid classification model is what we want. Gene expression programming (GEP) introduced by Ferreira [14] is an automatic programming approach, which overcomes certain limitations of genetic algorithms and genetic programming by working with two elements, the chromosome and the expression tree [15]. The advantage of GEP in designing decision trees makes it a successful method for solving classification problems [16,17]. Each physical and chemical parameter of AAs is as a gene unit in the gene expression programming. Complex algorithms weave them to a multivariate nonlinear equation. GEP in the field of carcinogenic classification shows incomparable superiority. The multilayer perceptrons (MLPs) is a biologically inspired computational tool for solving pattern recognition problems and is efficient in recognizing previously trained patterns. The capability of neural networks with multiple inputs and multiple outputs realizes data parallel processing and self-learning $[13,18]$. The parameters, as well as neurons, perform math functions intended to interweave them to a net, divided into carcinogens and non-carcinogens. In the current research, GEP and MLPs are new analysis strategies of the classification for carcinogenicity of AAs. Compared with MLPs, the proposed GEP is better in carcinogenic potency prediction of a suite of AA samples.

\section{Methodology}

\subsection{Source of AA Data}

25 compounds have ionic pentavalent nitrogen atoms, and hexavalent sulfur atoms were eliminated, because the physical and chemical parameters cannot be computed, 128 fused ring aromatic amine (including heterocyclic compound) were taken from the literature [19], molecular structures and data of carcinogenicity are available. 1 stands for carcinogen, 0 stands for non-carcinogen. Carcinogenic activity is indicated by rat liver tumor. In this study, random allocation was taken to assure that every compound has the same opportunity to be divided into training set and test set. Each compound was given to a encoding, from 1 to 128 . Then, 35 random numbers are generated in IBM SPSS 19.0 software (IBM Corporation, Chicago, IL, USA). If the encoding is same with the random number, this compound will be selected to test set. Finally, 128 compounds were divided into 93 training sets (Table 1) and 35 test sets (Table 2). The test set is used to evaluate stability of the QSAR model. 
Table 1. Carcinogenic activity of aromatic amines for training set.

\begin{tabular}{|c|c|c|c|c|}
\hline No. & Aromatic Amines & $\begin{array}{l}\text { Carcinogenicity } \\
\text { (exp) }\end{array}$ & $\begin{array}{l}\text { Carcinogenicity } \\
\text { (GEP) }\end{array}$ & $\begin{array}{l}\text { Carcinogenicity } \\
\text { (MLPs) }\end{array}$ \\
\hline 1 & $\mathrm{~N}$-Acetoxy-4-biphenylacetamide & 0 & 0 & 0 \\
\hline 2 & $\mathrm{~N}$-Acetoxy-2-fluorenylacetamide & 0 & 0 & 0 \\
\hline 3 & $N$-Acetoxy-4-phenanthrylacetamide & 0 & 0 & 0 \\
\hline 4 & $N$-Acetoxy- $N$-(4-stilbenyl)acetamide & 0 & 0 & 0 \\
\hline 5 & 3-Amino-s-triazole & 1 & 1 & 1 \\
\hline 6 & 1-Anthramine & 0 & 0 & 0 \\
\hline 7 & 9-Anthramine & 0 & 0 & 0 \\
\hline 8 & 2-Anthranilacetamide & 0 & 0 & 0 \\
\hline 9 & Benzidine & 1 & 0 & 1 \\
\hline 10 & $N$-(Benzoyloxy)-fluorenylacetamide & 0 & 1 & 0 \\
\hline 11 & 4-Biphenyldimethylamine & 0 & 0 & 0 \\
\hline 12 & 3,6-Bis(dimethylamino)acridine & 1 & 0 & 1 \\
\hline 13 & 2-Chloro-4-phenylaniline & 0 & 1 & 0 \\
\hline 14 & $4^{\prime}$-Chloro-4-stilbenyl- $N, N$-dimethylamine & 0 & 0 & 0 \\
\hline 15 & 2-Cyano-4-stilbenamine & 1 & 0 & 0 \\
\hline 16 & 4,6-Diamino-2-(5-nitro-2-furyl)-s-triazine & 0 & 1 & 1 \\
\hline 17 & $0,0^{\prime}$-Dianisidine & 0 & 0 & 0 \\
\hline 18 & 3-Dibenzofuranylacetamide & 0 & 0 & 0 \\
\hline 19 & 3-Dibenzothiophenylacetamide & 0 & 0 & 0 \\
\hline 20 & 2,2'-Dichloro-4,4'-diaminostilbene & 1 & 0 & 1 \\
\hline 21 & $3,3^{\prime}$-Dichloro-4, $4^{\prime}$-diaminostilbene & 0 & 1 & 0 \\
\hline 22 & 9,10-Dihydro-2-phenanthramine & 0 & 0 & 0 \\
\hline 23 & 3,3'-Dihydroxybenzidine & 0 & 0 & 0 \\
\hline 24 & 2-(4-(N,N-Dimethylamino)styryl) quinoline & 0 & 0 & 0 \\
\hline 25 & 3,2'-Dimethyl-4-biphenylamine & 0 & 0 & 0 \\
\hline 26 & 3,3'-Dimethyl-4-biphenylamine & 0 & 0 & 0 \\
\hline 27 & 2-Fluorenylacetamide & 1 & 0 & 0 \\
\hline 28 & 3-Fluorenylacetamide & 0 & 0 & 0 \\
\hline 29 & 1-Fluorenylaceto hydroxamic acid & 0 & 0 & 0 \\
\hline 30 & 2-Fluorenylaceto hydroxanic acid & 1 & 0 & 0 \\
\hline 31 & $N$-Fluorenyl-2-benzamide & 0 & 1 & 0 \\
\hline 32 & $N$-Fluorenyl-2-benzohydroxamic acid & 0 & 0 & 0 \\
\hline 33 & 2-Fluorenyldiacetamide & 1 & 0 & 1 \\
\hline 34 & 2-Fluorenyldimethylamine & 1 & 1 & 1 \\
\hline 35 & 2,5-Fluorenylenediacetamide & 0 & 0 & 0 \\
\hline 36 & 2-Fluorenylhydroxylamine & 0 & 0 & 0 \\
\hline 37 & $N$-(2-Fluorenyl)-2,2,2-trifluoroacetamide & 1 & 0 & 1 \\
\hline 38 & 4'-Fluoro-4-biphenylamine & 1 & 0 & 1 \\
\hline 39 & 1-Fluoro-2-fluorenylacetamide & 0 & 0 & 1 \\
\hline 40 & 3-Fluoro-2-fluorenylacetamide & 1 & 0 & 0 \\
\hline 41 & 4-Fluoro-2-fluorenylacetamide & 0 & 0 & 0 \\
\hline 42 & 5-Fluoro-2-fluorenylacetamide & 0 & 1 & 0 \\
\hline 43 & 6-Fluoro-2-fluorenylacetamide & 1 & 0 & 0 \\
\hline 44 & 7-Fluoro-2-fluorenylacetamide & 1 & 0 & 0 \\
\hline 45 & 7-Fluoro-2- $N$-fluorenylacetohydroxamic acid & 1 & 1 & 0 \\
\hline 46 & $4^{\prime}$-Fluoro- $p$-phenylaniline & 0 & 1 & 0 \\
\hline 47 & 4'-Fluoro-4-stilbenamine & 1 & 1 & 0 \\
\hline 48 & $4^{\prime}$-Fluoro-4-stilbenyl- $N, N$-dimethylamine & 1 & 0 & 0 \\
\hline 49 & 2-Hydrazino-4-phenylthiazole & 0 & 1 & 0 \\
\hline 50 & $N$-Hydroxy- $N$-(4-stilbenyl) acetamide & 0 & 1 & 0 \\
\hline 51 & 3-Iodo-2-fluorenylacetamide & 0 & 0 & 0 \\
\hline 52 & 7-Iodo-2-fluroenylacetamide & 0 & 0 & 0 \\
\hline 53 & 2-Methoxy-3-benzofuranylamine & 0 & 0 & 0 \\
\hline 54 & 7-Methoxy-2-fluorenylacetamide & 1 & 0 & 1 \\
\hline 55 & 1-Methoxy-2-fluorenylamine & 1 & 0 & 1 \\
\hline 56 & 3-Methoxy-2-fluorenylamine & 0 & 1 & 0 \\
\hline 57 & 4-((p-Methoxyphenyl)azo)-o-anisidine & 1 & 0 & 1 \\
\hline 58 & 2-Methyldiacetylbenzidine & 0 & 0 & 1 \\
\hline 59 & 4,4'-Methylenebis(2-chloroaniline) & 1 & 1 & 1 \\
\hline 60 & 4'-Methyl-4-phenylacetanilide & 0 & 0 & 0 \\
\hline
\end{tabular}


Table 1. Cont.

\begin{tabular}{|c|c|c|c|c|}
\hline No. & Aromatic Amines & $\begin{array}{l}\text { Carcinogenicity } \\
\text { (exp) }\end{array}$ & $\begin{array}{l}\text { Carcinogenicity } \\
\text { (GEP) }\end{array}$ & $\begin{array}{c}\text { Carcinogenicity } \\
\text { (MLPs) }\end{array}$ \\
\hline 61 & 3-Methyl-4-phenylaniline & 0 & 1 & 0 \\
\hline 62 & 3-Methyl-4-stilbenamine & 0 & 0 & 0 \\
\hline 63 & 1-Naphthylacetohydroxamic acid & 0 & 0 & 0 \\
\hline 64 & 2-Naphthylhydroxylamine & 0 & 0 & 0 \\
\hline 65 & 9-Oxo-2-fluorenylacetamide & 1 & 0 & 0 \\
\hline 66 & 1-Phenanthrylacetamide & 0 & 0 & 0 \\
\hline 67 & 2-Phenanthrylacetamide & 0 & 1 & 0 \\
\hline 68 & 1-Phenanthrylamine & 0 & 0 & 0 \\
\hline 69 & 3-Phenanthrylamine & 0 & 0 & 0 \\
\hline 70 & 9-Phenanthrylamine & 0 & 0 & 0 \\
\hline 71 & 4-(Phenylazo) acetanilide & 0 & 0 & 0 \\
\hline 72 & 4-(Phenylazo) aniline & 0 & 0 & 0 \\
\hline 73 & 4-(Phenylazo) diacetanilide & 0 & 0 & 0 \\
\hline 74 & 4-(Phenylazo)- $N$-phenylacetohydroxamic acid & 0 & 0 & 0 \\
\hline 75 & 4-Stilbenamine & 0 & 0 & 0 \\
\hline 76 & $N$-(4-Stilbenyl) acetamide & 0 & 0 & 0 \\
\hline 77 & 4-Stilbenyl- $N, N$-diethylamine & 0 & 0 & 0 \\
\hline 78 & 4-Stilbenyl- $N, N$-dimethylamine & 0 & 0 & 0 \\
\hline 79 & N-(4-Styrylphenyl) hydroxylamine & 0 & 0 & 0 \\
\hline 80 & $3,2^{\prime}, 4^{\prime}, 6^{\prime}$-Tetramethyl-4-biphenylamine & 1 & 0 & 0 \\
\hline 81 & $o, o^{\prime}$-Tolidine & 0 & 1 & 0 \\
\hline 82 & 4-(m-Tolylazo) acetanilide & 0 & 0 & 0 \\
\hline 83 & 4-(m-Tolylazo) aniline & 0 & 0 & 0 \\
\hline 84 & 2-(o-Tolylazo)- $p$-toluidine & 1 & 0 & 1 \\
\hline 85 & 2-( $p$-Tolylazo)- $p$-toluidine & 0 & 0 & 0 \\
\hline 86 & 4-(o-Tolylazo)-o-toluidine & 1 & 1 & 0 \\
\hline 87 & 4-(o-Tolylazo)-m-toluidine & 0 & 0 & 0 \\
\hline 88 & 4-(m-Tolylazo)-m-toluidine & 0 & 0 & 0 \\
\hline 89 & 4-( $p$-Tolylazo)-o-toluidine & 0 & 0 & 0 \\
\hline 90 & 4 -( $p$-Tolylazo)- $m$-toluidine & 0 & 0 & 0 \\
\hline 91 & $N, N, 2^{\prime}$-Trimethyl-4-stilbenamine & 0 & 0 & 0 \\
\hline 92 & $N, N, 3^{\prime}$-Trimethyl-4-stilbenamine & 0 & 0 & 0 \\
\hline 93 & $N, N, 4^{\prime}$-Trimethyl-4-stilbenamine & 0 & 0 & 0 \\
\hline
\end{tabular}

Table 2. Carcinogenic activity of aromatic amines for test set.

\begin{tabular}{ccccc}
\hline No. & Aromatic Amines & $\begin{array}{c}\text { Carcinogenicity } \\
(\mathbf{e x p})\end{array}$ & $\begin{array}{c}\text { Carcinogenicity } \\
\text { (GEP) }\end{array}$ & $\begin{array}{c}\text { Carcinogenicity } \\
\text { (MLPs) }\end{array}$ \\
\hline 1 & 2-Anthramine & 0 & 0 & 0 \\
2 & 4-Biphenylacetamide & 0 & 0 & 0 \\
3 & 4-Biphenylacetohydroxamic acid & 0 & 1 & 0 \\
4 & 3-Carbazolylacetamide & 0 & 0 & 1 \\
5 & 2,7-Diaminofluorene & 0 & 0 & 1 \\
6 & 4,4'-Diaminostilbene & 1 & 1 & 0 \\
7 & 2-Dibenzothiophenylacetamide & 0 & 0 & 0 \\
8 & 3,3'-Dichlorobenzidine & 0 & 0 & 0 \\
9 & 2-Fluorenamine & 1 & 1 & 0 \\
10 & 1-Fluorenylacetamide & 0 & 0 & 0 \\
11 & 3-Fluorenylaceto hydroxanic acid & 0 & 0 & 0 \\
12 & 2,7-Fluorenyldiacetamide & 1 & 1 & 0 \\
13 & 2-Fluorenyldiethylamine & 0 & 0 & 0 \\
14 & N,2-Fluorenylformamide & 0 & 1 & 0 \\
15 & 2-Fluorenylmethylamine & 1 & 0 & 1 \\
16 & N,2-Fluorenylsuccinamic acid & 1 & 0 & 0 \\
17 & 8-Fluoro-2-fluorenylacetamide & 1 & 0 & 0 \\
18 & 2-Fluoro-4-phenylaniline & 0 & 0 & 1 \\
19 & 3'-Fluoro-4-phenylaniline & 0 & 0 & 0 \\
20 & 3-Methoxy-4-biphenylamine & 0 & 1 & 0 \\
\hline
\end{tabular}


Table 2. Cont.

\begin{tabular}{ccccc}
\hline No. & Aromatic Amines & $\begin{array}{c}\text { Carcinogenicity } \\
\text { (exp) }\end{array}$ & $\begin{array}{c}\text { Carcinogenicity } \\
\text { (GEP) }\end{array}$ & $\begin{array}{c}\text { Carcinogenicity } \\
\text { (MLPs) }\end{array}$ \\
\hline 21 & 3-Methoxy-2-fluorenylacetamide & 0 & 1 & 0 \\
22 & 4,''-Methylenebis(2-methylaniline) & 1 & 0 & 1 \\
23 & 3-Methyl-2-naphthylamine & 0 & 0 & 0 \\
24 & 2-Methyl-4-phenylaniline & 0 & 0 & 0 \\
25 & 2'-Methyl-4-phenylaniline & 0 & 0 & 0 \\
26 & 2-Methyl-4-stilbenamine & 0 & 1 & 0 \\
27 & 2-Naphthylamine & 0 & 0 & 0 \\
28 & 1-Naphthylhydroxylamine & 0 & 0 & 0 \\
29 & 9-Phenanthrylacetamide & 0 & 0 & 0 \\
30 & 2-Phenanthrylacetohydroxamic acid & 0 & 0 & 0 \\
31 & 2-Phenanthrylamine & 0 & 1 & 0 \\
32 & 4-(Phynylazo)-o-anisidine & 1 & 1 & 0 \\
33 & 1-(Phenylazo)-2-naphthylamine & 0 & 0 & 0 \\
34 & 4-(Phenylazo)- $N$-phenylhydroxylamine & 0 & 0 & 1 \\
35 & 3,2', '5'-Trimethyl-4-diphenylamine & 1 & 0 & \\
\hline
\end{tabular}

\subsection{Calculation of Molecular Descriptors}

In the QSAR model, molecular structure of compound was replaced by the corresponding physical and chemical parameters to establish numerical equations. All the structures of AAs were drawn into Chemdraw. Firstly, the geometry optimization operated in the Hyperchem 7.5 software (HyperCube Inc., Gainesville, FL, USA), the calculation used MM+ molecular mechanics force field. The semi-empirical AM1 method can get more precise optimization in the MOPAC. The molecular structures were optimized using the Polak-Ribiere algorithm until the root mean square gradient was 0.01 [20]. Then, the HIN files were generated by geometrical optimization, the MNO files were generated by MOPAC calculation. The CODESSA program (Semichem, Shawnee, KS, USA) can give the five classes of descriptors: constitutional, topological, geometrical, electrostatic, and quantum-chemical. Semi-empirical quantum chemistry methods are on the basis of the Hartree-Fock formalism, but include some approximations and obtain some parameters from empirical data. They are very appropriate for computational chemistry for treating physicochemical properties of large molecules. The semi-empirical AM1 calculation has shown to be successful in studying of QSAR. The constitutional descriptors show the molecular composition of the compounds without using the geometry or electronic structure; including number of atoms, molecular weight, and average atomic weight, etc. The topological descriptors are used to describe the atomic connectivity in the molecule; including wiener index, information content index and its derivatives, etc. The geometrical descriptors provide the information about the size of the molecule and require 3D-coordinates of the atoms in the given molecule; including shadow indices, molecular volume, etc. The electrostatic descriptors can reflect characteristics of the charge distribution of the molecule; including charged partial surface area descriptors, partial positive surface area, etc. The quantum-chemical descriptors will add important information to the conventional descriptors; including HOMO-LUMO energy gap, reactivity indices, etc. With the method of preprocessing, according to the following three rules [21], the model necessary descriptors are selected: (1) The parameters are the common of vast majority of the compounds; (2) For all the compounds, the descriptor numerical decreases; (3) The correlation coefficient of any two variables should $<0.8$. If any two descriptors have a correlation of 0.8 , one should be removed. Otherwise, it will reduce the prediction efficiency of the QSAR model. This method could be used using well-established statistical projection technique such as PLS [22] or ACP [23] to construct uncorrelated variables.

\subsection{Theory of Gene Expression Programming}

Gene expression programming (GEP) is a new technique of novel algorithm for data mining that is based on the structure and function of biological gene [24]. It carries on all the advantages of both 
genetic algorithm (GA) and genetic programming (GP), by eliminating some of their own limitations. GEP adopts fixed length, nonlinear, or linear strings of chromosomes to solve complex problems by forming the expression trees of different shapes and sizes when evaluating their fitness [25]. The search space of GEP is separated from the solution space, which can be expanded to the benefits such as unconstrained search of the genome space, thus achieving the purpose of using simple coding to solve classification problems.

GEP genotype individuals consist of the head and tail, the head elements from the function character and terminator sets, tail elements from terminator sets. The head is not strictly limited. The length of the head $h$ is selected according to the number of parameters (such as a, b, c, 1, 2 . ) and the set of functions (such as $\sin , \tan \ldots$ ). The common set of functions, $F=\{+,-, *, \div, Q\}$, $Q$ represents the root function. The tail only contains the variable. The length of the tail $t$ should be computed as: $t=h(n-1)+1$. $n$ is the number of parameters for the maximum number variable function. The chromosomes function as a genome, after being modified by various means of mutation, transposition, root transposition, gene transposition, gene recombination, and one-point and two-point recombination, that will be transformed into expression trees. Figure 1 is one of the simplest expression trees can be processed into QSAR formula: $F=b *(a+(c-\sqrt{d}))$. Parameters in the operation relationship were used to set up various models until get the best results. The application of complex functions can improve the prediction ability of QSAR model.

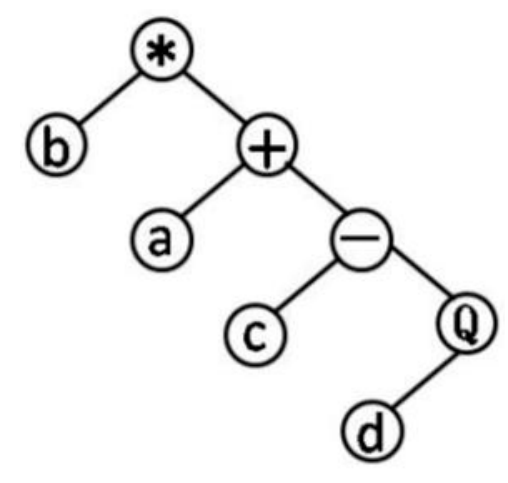

Figure 1. Expression trees.

It is important that individuals to be selected and copied into the next generation according to the fitness function. The advantage of this kind of fitness function is the system that can find the optimal solution for itself. The calculation [26] for optimum fitness function (Equations (1)-(3)):

$$
\begin{gathered}
\text { fitness }(R)= \begin{cases}0, & \text { if consig }(R)<0 \\
\operatorname{consig}(R) * \ln (\operatorname{compl}(R)-1), & \text { otherwise }\end{cases} \\
\operatorname{consig}(R)=\left(\frac{p}{p+n}-\frac{P}{P+N}\right) * \frac{P}{P+N} \\
\operatorname{compl}(R)=\frac{p}{P}
\end{gathered}
$$

$p, n, P$, and $N$ are number of all the positive compounds, number of all the negative compounds, number of the positive compounds in a training set, and number of the negative compounds in a training set, respectively.

For two classification prediction problems, only one GEP rule classification $(R)$ can be achieved. Validating instances with GEP rules, if the result is positive, will be considered as a kind of the instance. Otherwise, it should be to the other kind. Exact representation is as follows: 
If GEP_Rule $(X)>0$ Then $X \in$ class $A$

ELSE $X \in$ class $B$

$X$ stands for properties of instance.

The process of classification prediction problem is that decoding and calculating the fitness function of each chromosome, performing all kinds of genetic operation and updating chromosomes. This process will be repeated for a pre-established number of generations until the best model has been found [20]. Flow chart of GEP classification algorithm is shown in Figure 2.

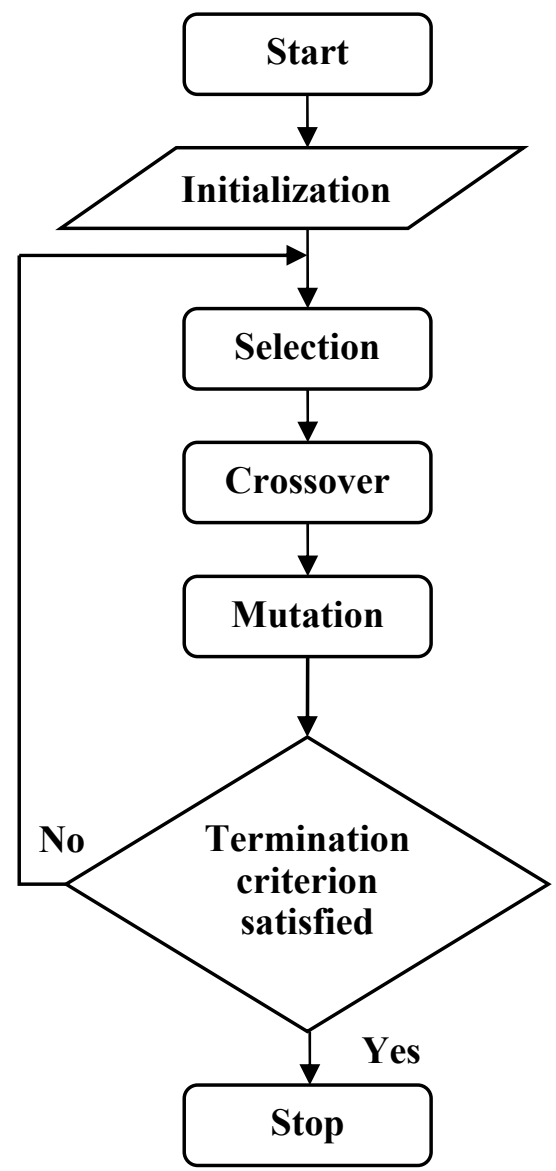

Figure 2. The flow chart of GEP.

\subsection{Multilayer Perceptrons (MLPS)}

Artificial neural network (ANN) is based on the structure and function of neural network. It puts the complex neural network theory to simplify, abstract, and simulate. ANN has been widely used in classification, prediction, associative memory, pattern recognition, and other fields, which has gotten consistently high praise. What makes a MLPs different is that some neurons use a nonlinear activation function which is developed to model the frequency of action potentials, or firing, of biological neurons in the brain. Weka software provides a multilayer perceptrons artificial neural network. The use of back-propagation network algorithms makes MLPs application more expansive than other artificial neural networks. Figure 3 shows the structure of MLPs. 


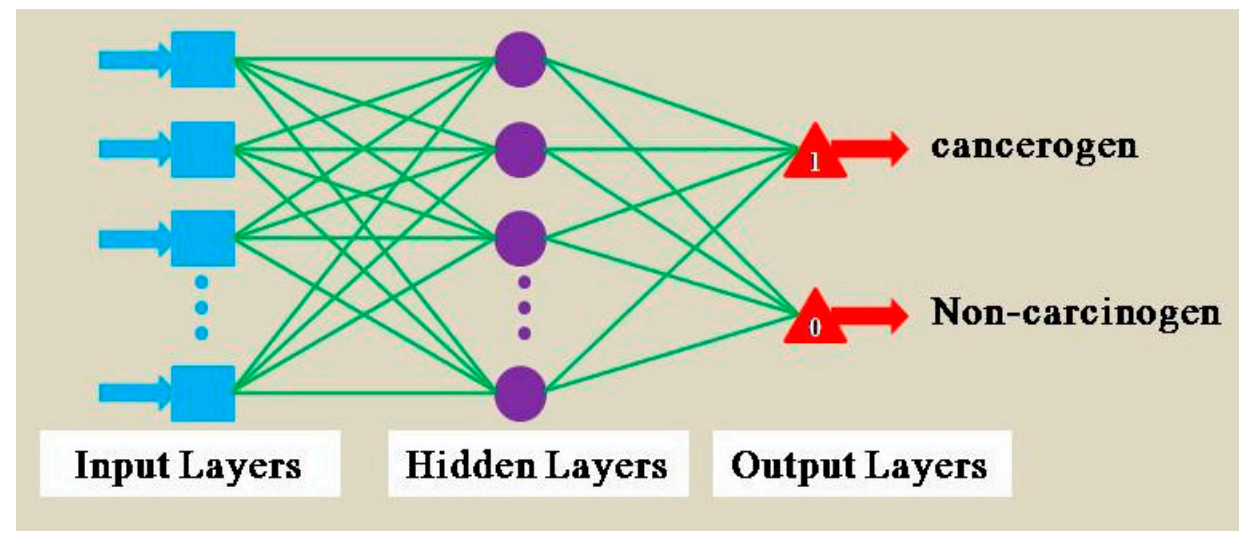

Figure 3. Multilayer perceptrons artificial neural network structure.

The input layer is decided by the dimensions of objects and the received signal is directly transmitted to the hidden layers. The number of hidden layers cannot be calculated by an accurate analytical formula and usually determined according to experience. In Weka, universal symbol "a" represents for hidden layers, $a=\frac{\text { attribs }+ \text { classes }}{2}$. The realization of signal transmission and output of nodes between hidden layer and output layer is by excitation function [27]. Basic idea of carcinogenic classification forecasting of AAS by MLPs is that the known results of the sample model used for training network, and the carcinogenicity of compounds, can be identified by the trained network.

\subsection{Platform of Weka}

Waikato Environment for Knowledge Analysis (Weka) was developed by IanH. Wjttjn and EibeFrank of the University of Waikato and was based on JAVA software. As professional data mining software, Weka contains almost all of the classification methods in machine learning [28,29]. Under normal circumstances, the scholars are unable to preprocess the complex data without a good data analysis background of data mining. Weka provides a unified interface for users and saves manual programming for data analysis. Weka can not only provide a single classification algorithm of projections for the same data, but also can integrate several algorithms to predicting. To our knowledge, the rationale and complexity of classification algorithms can affect the accuracy of the prediction. Therefore, we chose different algorithm and combined the test of GEP and MLPs, so that we can obtain better prediction results and provide a good model.

\section{Results and Discussion}

\subsection{Significance of the Descriptors}

Number of carbon atoms (NCOS): The number of benzene rings is associated with the carcinogenicity. Growing number of $C$ atoms will increase the morbidity of cancer [30]. On the other hand, the binding of methyl with DNA can change the conformation of double helix and affect the transcription of protein, which then changes tumor suppressor genes and gene mutation increases the risk of cancer [31]. The number of $C$ atoms in nitrobenzene as descriptors to build the QSAR model has important significance.

Number of nitrogen-atoms (NNOS): Aromatic amines metabolic activation sites on the amino $\mathrm{N}$ atoms. Preliminary metabolic activation occurs in the liver, including $\mathrm{N}$-catalytic oxidation by cytochrome P4501A2 and $N$-acetylation by acetyl enzyme catalysis. This process produce $N$-hydroxy. The aryl amines generated from oxidation can form additions with DNA to the urinary tract epithelial cells. Likewise, the structure of DNA is changed. $\mathrm{N}$-O-sulfate ester is formed after sulfur transfer with $\mathrm{N}$-hydroxyl. Another way, the reaction of $\mathrm{N}$-hydroxyl with acetyltransferase produces $\mathrm{N}$-O -acetate ester. The unstable $\mathrm{N}$-O-sulfate ester and $\mathrm{N}$-O-acetate ester generate $\mathrm{N}$ ions in hydrolysis, which can 
combine with normal ion-making nucleophilic reaction with DNA bases [30,32]. Highly activated free radical nitrogen ions cause normal cell mutation.

Kier flexibility index (KFBI), Balaban index (BBI), structural information content index (order 0) (SICI), and topographic electronic index (all bonds) (TEIA) are topology descriptors. The molecular connectivity index as the structure characterization can provide a intuitive concept to make quantitative description on the molecular structure according to the molecular size, shape, and structure of chemical bond connection sequence and branched molecules-such as the structure of the information. The topology descriptors make structural differences quantitatively between the molecular quantitative and expression of molecular connectivity function. Different numerical topology values represent different molecular structures [32-34]. The four kinds of molecular descriptors are closely connected with carcinogenicity of AAs and can be used for the QSAR model.

Polarity parameter (PLPT) is closely related to the solubility of molecules. The larger the lipo-hydro partition coefficient of low polar compounds, the higher the lipid solubility. It easily gets the lipid bilayer by simple diffusion and accumulates in adipose tissue. High polar compounds have better water-solubility. Water-solubility directly affects the toxicity and the target organ [35]. The polarity of aromatic amine determines the metabolism time in the body. The polarity parameters as discriminant factors are very crucial.

The lower the LUMO energy (LUMO) is more conducive to electrophilic reaction. Electrophilic reagents are related to the carcinogenicity of AA compounds. AAs could be converted to electrophilic reagents that are with some or all of the positive charge under the effect of cytochrome P-450 or other oxidase [36]. The atom with electrons in nucleophilic reagent easily reacts with the electrophilic reagents by sharing electrons. AAs as a promoter can enhance the carcinogenic effect of other poisons.

The correlation of eight descriptors is calculated by SPSS 20.0, in which any two variables related factor $<0.8$ (Table 3). It means that all variables are uncorrelated and not repetitive in the GEP models, so all the eight parameters could be adapted to QSAR study.

Table 3. The correlation of eight descriptors.

\begin{tabular}{|c|c|c|c|c|c|c|c|c|}
\hline Correlation & NCOS & NNOS & KFBI & BBI & SICI & TEIA & PLPT & LUMO \\
\hline NCOS & 1.000 & -0.227 & 0.649 & -0.708 & 0.667 & 0.234 & -0.034 & -0.374 \\
\hline NNOS & & 1.000 & 0.175 & -0.014 & 0.159 & 0.312 & -0.201 & -0.111 \\
\hline KFBI & & & 1.000 & -0.569 & 0.730 & 0.433 & 0.007 & -0.18 \\
\hline BBI & & & & 1.000 & -0.681 & -0.259 & -0.173 & 0.438 \\
\hline SICI & & & & & 1.000 & 0.620 & 0.250 & -0.456 \\
\hline TEIA & & & & & & 1.000 & 0.339 & -0.107 \\
\hline PLPT & & & & & & & 1.000 & -0.277 \\
\hline LUMO & & & & & & & & 1.000 \\
\hline
\end{tabular}

\subsection{Results of GEP}

128 compounds include 35 carcinogenic and 93 non carcinogenic. The number of carcinogenic and non-carcinogenic compounds is 24 , with 64 in the training set, respectively. The setting of the function is $\{+,-, \times, \div, \operatorname{Mod}$, Exp, Log, $\operatorname{Sin}$, Tan $\}$, eight groups descriptors were used to build GEP model in the Automatic Problem Solver 3.0 (Gepsoft Limited Company, Bristol, UK). It takes about 25 min to select a most optimal model. Prediction result of each compound, accuracy, positive predictive value and negative predictive value (Table 4 ) are given by APS. We converted the $\mathrm{C}++$ function into Equation (4).

$$
\begin{aligned}
& F(x)=x_{1}+\tan \left[\log \left(x_{8}+x_{5}\right)-\frac{x_{1} \cdot x_{6}}{x_{3}+x_{2}}\right] \\
& +\frac{\tan \left(x_{5}+x_{3}\right)}{\bmod \left[\log \left(x_{6} \cdot x_{2}\right), \log x_{8}\right]}+x_{5}+ \\
& \tan \left(x_{2}+x_{3}+x_{7}-x_{1}\right)+\tan \left(x_{4}+x_{8}-x_{1}\right) \\
& +\tan \left\{\exp \left[\log \left(\frac{x_{5}}{\bmod \left(x_{3}, x_{1}\right)}\right)+x_{5}\right]\right\}
\end{aligned}
$$


The variables $x_{1}, x_{2}, x_{3}, x_{4}, x_{5}, x_{6}, x_{7}$, and $x_{8}$ represents the Number of $C$ atoms, Number of $\mathrm{N}$ atoms, Kier flexibility index, Balaban index, structural information content index (order 0), and topographic electronic index (all bonds), polarity parameter, and the lower LUMO energy.

This is a complex nonlinear function, but classification prediction result is pretty better. Accurate rates of training set and test set are 0.92 and 0.82 .

Table 4. Results of GEP and MLPs.

\begin{tabular}{ccccc}
\hline & Accuracy & Sensitivity & Specificity & Youden's Index \\
\hline Training set of GEP & 0.914 & 0.947 & 0.905 & 0.852 \\
Test set of GEP & 0.829 & 0.667 & 0.885 & 0.552 \\
Training set of MLPS & 0.838 & 0.844 & 0.813 & 0.657 \\
Test set of MLPS & 0.743 & 0.793 & 0.500 & 0.293 \\
\hline
\end{tabular}

\subsection{The Results of MLPs}

Hidden layers were set "a", training time is 500, validation threshold is 20 . The test set is same with that of GEP. The training set is used to adjust the parameters of the model, and the test set is used to evaluate the predictive power. MLPs use the back-propagation algorithm and keep regulating weights in training to get the global error minimized.

The entire range of carcinogenic aromatic amine prediction accuracy is 0.84 of training set and 0.74 of test set by MLPs. Grid square represents error prediction. Curve margin could intuitively reflect the quality of classification prediction results. Curve margin is the difference values of forecasting, probability of actual categories, and the maximum prediction probability of wrong categories. The vertical axes represent the sequence numbers of AAs. The horizontal axes represent the difference values. The greater difference values of samples closer to 1 , the better classification effect. Figures 4 and 5 show the vast majority of marginal values are close to 1 . These two pictures indicate MLPs can accurately predict the carcinogenicity of AAs. The results of MLPs are given by Weka (Table 4). From the point of view of running time, $0.08 \mathrm{~s}$ for training set and $0.20 \mathrm{~s}$ for the test set.

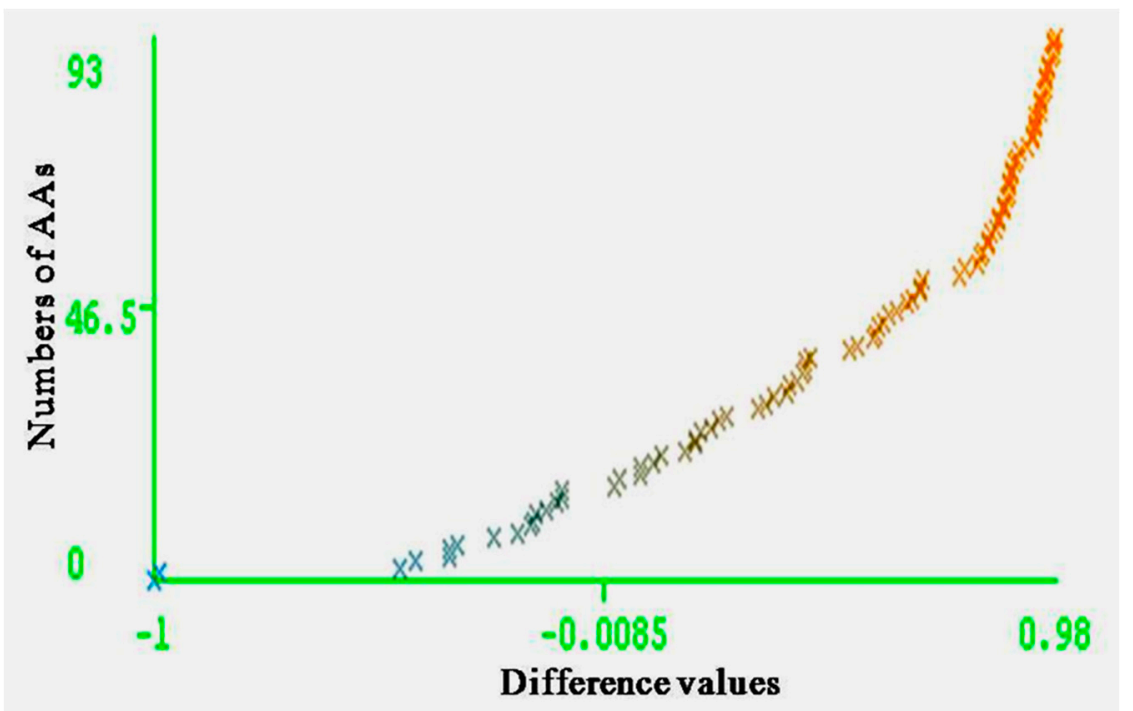

Figure 4. Curve margin of training set. The vertical axes represent the numbers of AAs; the horizontal axes represent the difference values of forecasting probability of actual categories, and the maximum prediction probability of wrong categories. 


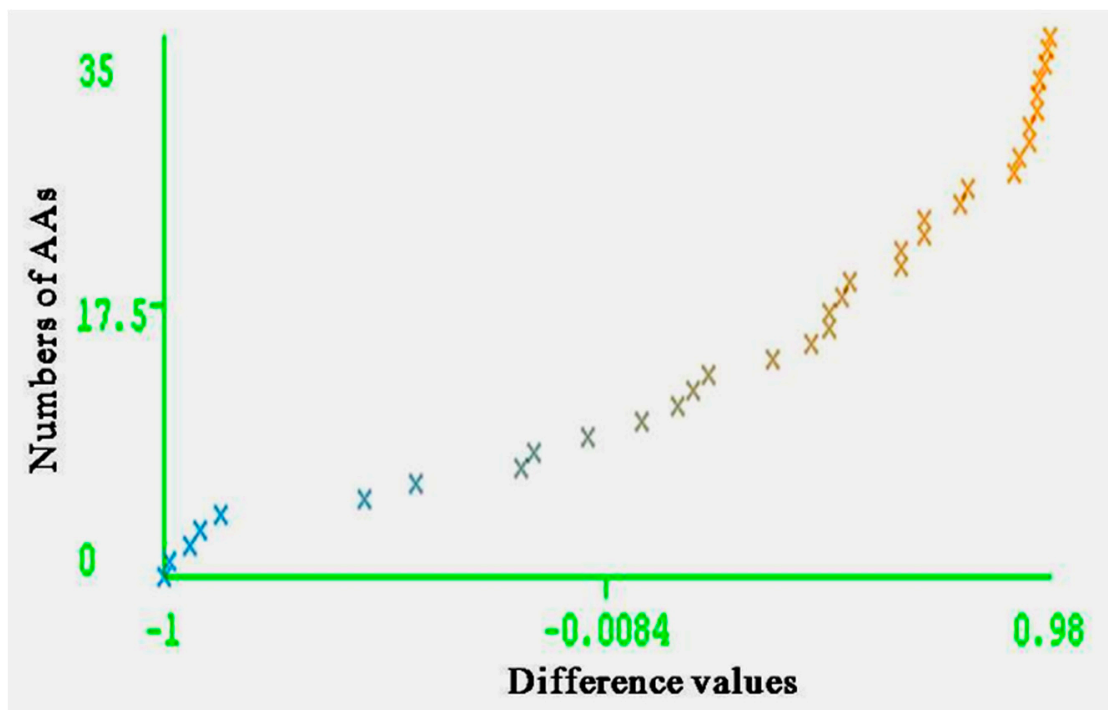

Figure 5. Curve margin of test set. The vertical axes represent the numbers of AAs; the horizontal axes represent the difference values of forecasting probability of actual categories and the maximum prediction probability of wrong categories.

\subsection{Comparison between GEP and MLPS}

The purpose of this study is to establish a precise prediction model, to accurately identify the potential carcinogen of AAs. Carcinogenic compound prediction is very rare in previous studies. The GEP model based on human gene expression could accurately identify the carcinogenic of AAs. Performance assessment of classification algorithm shown in Table 4 uses recognized indicators precision, sensitivity, specificity, and Youden's index obtained by optimizing Equations (5)-(7). These indexes are cited from "screening test" of epidemiology. Screening test has been widely employed in seeking potential patients to provide medical help in time. The indexes (accuracy, sensitivity, specificity, and Youden's index) can show the reliability of screening tests. Our study combined QSAR and screening test methods from epidemiology.

$$
\begin{gathered}
\text { sensitivity }=\frac{A}{A+C} \\
\text { specificity }=\frac{D}{B+D} \\
\text { Youden's index }=(\text { sensitivity }+ \text { specificity })-1
\end{gathered}
$$

where A and B are the number of carcinogenic compounds predicted correctly and wrongly by QSAR model, $C$ and $D$ are the number of non-carcinogenic compounds predicted wrongly and correctly by QSAR model, respectively. All these indexes are cited from screening of epidemiology.

GEP is significantly better than MLPs. This is mainly because GEP algorithm could construct adaptive function by the evolution of its own and establish nonlinear relationship between the details and the carcinogenic compounds. Due to unique way of coding and genetic operation, GEP possesses remarkable ability to predict the carcinogenicity of AAs. So that the GEP algorithm will be more details to reflect differences in the resulting expression. GEP can give detailed predicted expressions while MLPs only provide prediction results. However, the GEP model is a complex nonlinear function and in the process of the establishment of the model is full of complications.

MLPs in the study results are not as good as GEP. To get a satisfactory result, GEP often taking a long time, but the MLP run time is within one second. For MLPs, there are no universal common rules specify how to set up training methods, build network structure and select the parameters. It adopted 
"trial and error" that large amounts of neural networks were tested until an optimal result was obtained. The network structure and parameter settings are usually through personal experience [37]. In addition, MLPs cannot accurately reflect the nonlinear relationship between multiple parameters. MLP are only used for existing AAs carcinogenicity data, but it cannot establish equation expressions to predict the properties of new compounds. The probability is obtained among the independence of each various property, but in practice this is not the case, it may lead to a decline precise rate. MLPs cannot give mathematical expression of the model. Although the computational time of GEP is much more than MLPs, the forecasting accuracy is more important within a certain range (computational time not too long).

\section{Conclusions}

The study on the carcinogenic compounds of compounds is essential in toxicology. The structure of the chemical compounds is the basis for the toxicity and effect the metabolism of toxic chemicals in the body. QSAR is an innovative idea to predict the carcinogenicity of AAs. QSAR can evaluate the superiority of the experimental group and give the valuable information for the risk assessment. In this study, the computational time of MLPs is lower than GEP, but the forecasting ability of GEP is better than MLPs. The unique advantage of GEP is that it can establish a mathematical model to predict the toxicity of new compound. In the design of AA compounds, it can increase or reduce the certain structure to achieve the purpose of reducing carcinogenic potential. Thus, GEP is a promising research direction in toxicology.

Author Contributions: Fucheng Song, Anling Zhang, Hui Liang and Lianhua Cui conceived and designed the experiments; Fucheng Song performed the experiments; Wenlian Li and Hongzong Si analyzed the data; Yunbo Duan and Honglin Zhai contributed reagents/materials/analysis tools; Fucheng Song and Hongzong Si wrote the paper. All authors read and approved the final manuscript.

Conflicts of Interest: The authors declare no conflict of interest.

\section{References}

1. Harding, A.P.; Popelier, P.L.A.; Harvey, J.; Giddings, A.; Foster, G.; Kranz, M. Evaluation of aromatic amines with different purities and different solvent vehicles in the Ames test. Regul. Toxicol. Pharm. 2015, 71, 244-250. [CrossRef] [PubMed]

2. Garrigós, M.C.; Reche, F.; Marín, M.L.; Pernías, K.; Jiménez, A. Optimization of the extraction of azo colorants used in toy products. J. Chromatogr. A 2002, 963, 427-433. [CrossRef]

3. Sanchis, Y.; Coscollà, C.; Roca, M.; Yusà, V. Target analysis of primary aromatic amines combined with a comprehensive screening of migrating substances in kitchen utensils by liquid chromatography-high resolution mass spectrometry. Talanta 2015, 138, 290-297. [CrossRef] [PubMed]

4. Ewald, R.; Thomas, M.; Joerg, D.; Lynda, C.; Regina, S. Heterocyclic aromatic amines and their contribution to the bacterial mutagenicity of the particulate phase of cigarette smoke. Toxicol. Lett. 2016, 243, 40-47.

5. Wellner, T.; Lüersen, L.; Schaller, K.H.; Angerer, J.; Xler, H.; Korinth, G. Percutaneous absorption of aromatic amines-A contribution for human health risk assessment. Food Chem. Toxicol. 2008, 46, 1960-1968. [CrossRef] [PubMed]

6. Inami, K.; Okazawa, M.; Mochizuki, M. Mutagenicity of aromatic amines and amides with chemical models for cytochrome p450 in Ames assay. Toxicol. In Vitro 2009, 23, 986-991. [CrossRef] [PubMed]

7. Akceylan, E.; Bahadir, M.; Yllmaz, M. Removal efficiency of a calix[4]arene-based polymer for water-soluble carcinogenic direct azo dyes and aromatic amines. J. Hazard. Mater. 2009, 162, 960-966. [CrossRef] [PubMed]

8. Bratberg, M.; Olsvik, P.A.; Edvardsen, R.B.; Brekken, H.K.; Vadla, R.; Meier, S. Effects of oil pollution and persistent organic pollutants (POPs) on glycerophospholipids in liver and brain of male Atlantic cod (Gadus morhua). Chemosphere 2013, 90, 2157-2171. [CrossRef] [PubMed]

9. Merwe, J.P.V.D.; Hodge, M.; Olszowy, H.A.; Whittier, J.M.; Lee, S.Y. Using blood samples to estimate persistent organic pollutants and metals in green sea turtles (Chelonia mydas). Mar. Pollut. Bull. 2010, 60, 579-588. [CrossRef] [PubMed] 
10. Sondra, S.T.; Corrella, S.D. The biomechanisms of metal and metal-oxide nanoparticles' interactions with cells. Int. J. Environ. Res. Public Health 2015, 12, 1112-1134.

11. Sama, A.; Ayoub, K.J. Verifying the performance of artificial neural network and multiple linear regression in predicting the mean seasonal municipal solid waste generation rate: A case study of Fars province, Iran. Waste Manag. 2016, 48, 14-23.

12. Li, Y.W.; Shi, X.L.; Zhang, Q.Z.; Hu, J.T.; Chen, J.M.; Wang, W.X. Computational evidence for the detoxifying mechanism of epsilon class glutathione transferase toward the insecticide DDT. Environ. Sci. Technol. 2014, 48, 5008-5016. [CrossRef] [PubMed]

13. Uysala, M.; Tanyildizi, H. Estimation of compressive strength of self compacting concrete containing polypropylene fiber and mineral additives exposed to high temperature using artificial neural network. Constr. Build. Mater. 2012, 27, 404-414. [CrossRef]

14. Ferreira, C. Gene expression programming: A new adaptive algorithm for solving problems. Complex Syst. 2001, 1, 87-129.

15. Shaw, A.K.; Majumder, S.; Sarkar, S.; Sarkar, S.K. A novel EMD based watermarking of fingerprint biometric using GEP. Procedia Technol. 2013, 10, 172-183. [CrossRef]

16. Prasenjit, D.; Ajoy, K.D. A utilization of GEP (gene expression programming) meta model and PSO (particle swarm optimization) tool to predict and optimize the forced convection around a cylinder. Energy 2016, 95, 447-458.

17. Jędrzejowicz, J.; Jędrzejowicz, P. Experimental evaluation of two new GEP-based ensemble classifiers. Expert Syst. Appl. 2011, 38, 10932-10939. [CrossRef]

18. Silva, A.A.; Lima Neto, I.A.; Misságia, R.M.; Ceia, M.A.; Carrasquilla, A.G.; Archilha, N.L. Artificial neural networks to support petrographic classification of carbonate-siliciclastic rocks using well logs and textural information. J. Appl. Geophys. 2015, 117, 118-125. [CrossRef]

19. Zhu, Y.P.; Yu, Y.N.; Chen, X.R. Fisher discriminant analysis for carcinogenic potency of aromatic amines. Chin. J. Prev. Med. 1999, 1, 1-11.

20. Si, H.Z.; Wang, T.; Zhang, K.J.; Hu, Z.D.; Fan, B.T. QSAR study of 1,4-dihydropyridine calcium channel antagonists based on gene expression programming. Bioorganic Med. Chem. 2006, 14, 4834-4841. [CrossRef] [PubMed]

21. Li, X.Y.; Luan, F.; Si, H.Z.; Hu, Z.D.; Liu, M.C. Prediction of retention times for a large set of pesticides or toxicants based on support vector machine and the heuristic method. Toxicol. Lett. 2007, 175, 136-144. [CrossRef] [PubMed]

22. Servien, R.; Mamy, L.; Li, Z.; Rossard, V.; Latrille, E.; Bessac, F.; Patureau, D.; Benoit, P. Typol—A new methodology for organic compounds clustering based on their molecular characteristics and environmental behavior. Chemosphere 2014, 111, 613-622. [CrossRef] [PubMed]

23. Zhou, C.; Xiao, W.; Tirpak, T.M.; Nelson, P.C. Evolving Accurate and Compact Classification Rules with Gene Expression Programming. IEEE Trans. Evol. Comput. 2003, 7, 519-531. [CrossRef]

24. Eriksson, L.; Andersson, P.L.; Johansson, E.; Tysklind, M. Megavariate analysis of environmental QSAR data. Part I-A basic framework founded on principal component analysis (PCA), partial least squares (PLS), and statistical molecular design (SMD). Mol. Divers. 2006, 10, 169-186. [CrossRef] [PubMed]

25. Duan, L.; Tang, C.; Zhang, T.; Wei, D.; Zhang, H. Distance guided classification with gene expression programming. Adv. Data Min. Appl. 2006, 4093, 239-246.

26. Teodorescu, L.; Sherwood, D. High energy physics event selection with gene expression programming. Comput. Phys. Commun. 2008, 178, 409-419. [CrossRef]

27. Yadav, A.K.; Malik, H.; Chandel, S.S. Selection of most relevant input parameters using WEKA for artificial neural network based solar radiation prediction models. Renew. Sust. Energy Rev. 2014, 31, 509-519. [CrossRef]

28. Mohammad, M.N.; Sulaiman, N.; Muhsin, O.A. A novel intrusion detection system by using intelligent data mining in WEKA environment. Procedia Comput. Sci. 2011, 3, 1237-1242. [CrossRef]

29. Lievens, S.; Baets, B.D. Supervised ranking in the WEKA environment. Inf. Sci. 2010, 180, 4763-4771. [CrossRef]

30. Singh, K.P.; Gupta, S.; Rai, P. Identifying pollution sources and predicting urban air quality using ensemble learning methods. Atmos. Environ. 2013, 80, 426-437. [CrossRef] 
31. Kar, S.; Roy, K. First report on development of quantitative interspecies structure-carcinogenicity relationship models and exploring discriminatory features for rodent carcinogenicity of diverse organic chemicals using OECD guidelines. Chemosphere 2012, 87, 339-355. [CrossRef] [PubMed]

32. Wu, X.C.; Zhang, Q.Z.; Wang, H.; Hu, J.T. Predicting carcinogenicity of organic compounds based on CPDB. Chemosphere 2015, 139, 81-90. [CrossRef] [PubMed]

33. Helguera, A.M.; Pérez, M.A.C.; González, M.P.; Ruiz, R.M.; Díaz, H.G. A topological substructural approach applied to the computational prediction of rodent carcinogenicity. Bioorganic Med. Chem. 2005, 13, 2477-2488. [CrossRef] [PubMed]

34. Basavaraja, J.; Inamdar, S.R.; Kumar, H.M.S. Solvents effect on the absorption and fluorescence spectra of 7-diethylamino-3-thenoylcoumarin: Evaluation and correlation between solvatochromism and solvent polarity parameters. Spectrochim. Acta A 2015, 137, 527-534. [CrossRef] [PubMed]

35. Sambathkumar, K.; Jeyavijayan, S.; Arivazhagan, M. Electronic structure investigations of 4-aminophthal hydrazide by UV-visible, NMR spectral studies and HOMO-LUMO analysis byab initioand DFT calculations. Spectrochim. Acta A 2015, 147, 124-138. [CrossRef] [PubMed]

36. Lin, I.S.; Fan, P.L.; Chen, H.I.; Loh, C.H.; Shih, T.S.; Liou, S.H. Rapid and intermediate N-acetylators are less susceptible to oxidative damage among 4,4-methylenebis(2-chloroaniline) (MBOCA)-exposed workers. Int. J. Hydrogen Energy 2013, 216, 515-520. [CrossRef] [PubMed]

37. Szczuka, M.; Ślęzak, D. Feedforward neural networks for compound signals. Theor. Comput. Sci. 2011, 412, 5960-5973. [CrossRef]

(C) 2016 by the authors; licensee MDPI, Basel, Switzerland. This article is an open access article distributed under the terms and conditions of the Creative Commons Attribution (CC-BY) license (http://creativecommons.org/licenses/by/4.0/). 